\title{
Transient Neonatal Diabetes Mellitus in a Very Preterm Infant due to ABCC8 Mutation
}

\author{
Barbara Piccini, MD, $\mathrm{PhD}^{1}$ Caterina Coviello, $\mathrm{MD}^{2}$ Livia Drovandi, $\mathrm{MD}^{2}$ Artuso Rosangela, $\mathrm{MD}^{3}$ \\ Francesca Monzali, $\mathrm{BSc}^{4}$ Emilio Casalini, MD ${ }^{5}$ Sabrina Giglio, MD ${ }^{3,6}$ Sonia Toni, MD ${ }^{1}$ Carlo Dani, MD \\ ${ }^{1}$ Tuscany Regional Centre of Pediatric Diabetes, Meyer University \\ Children's Hospital, Florence, Italy \\ 2 Division of Neonatology, Careggi University Hospital of Florence, \\ Florence, Italy \\ ${ }^{3}$ Medical Genetics Unit, Meyer University Children's Hospital, \\ Florence, Italy \\ ${ }^{4}$ Pediatric Dietology Unit, Meyer University Children's Hospital, \\ Florence, Italy \\ ${ }^{5}$ University of Florence, Florence, Italy \\ ${ }^{6}$ Medical Genetics Unit, Department of Clinical and Experimental \\ Biomedical Sciences 'Mario Serio', University of Florence, Florence, Italy \\ 7 Department of Neuroscience, Psychology, Drug Research and Child \\ Health, Careggi University Hospital of Florence, Florence, Italy \\ Address for correspondence Carlo Dani, MD, Division of \\ Neonatology, Careggi University Hospital, 3 Largo Brambilla, \\ Florence, 50141, Italy (e-mail: cdani@unifi.it). \\ Am J Perinatol Rep 2018;8:e39-e42.
}

\author{
Abstract \\ Keywords \\ - neonatal diabetes \\ mellitus \\ - monogenic diabetes \\ - ABCC8 \\ - next-generation \\ sequencing \\ - $\beta$ cells \\ - newborns
}

Neonatal diabetes mellitus (NDM) is a monogenic form of diabetes occurring within 6 months from birth. NDM can be permanent or transient (TNDM). We report the case of a preterm infant with TNDM due to an ABCC 8 mutation identified by next-generation sequencing. The pancreatic adenosine triphosphate (ATP)-sensitive $\mathrm{K}+$ (K-ATP) channel is a key regulator of insulin secretion. Gain-of-function mutations in the genes encoding the Kir6.2 (KCNJ11) and SUR1 (ABCC8) subunits of the channel cause neonatal diabetes. The patient was successfully managed with insulin lispro at a 1:100 dilution, drawn up in an insulin pen injector with a 4-mm needle. The insulin lispro dilution allowed administration of the exact insulin doses, obtaining a good glycemic control and minimizing the burden of injections. At 2 months, corrected age insulin doses were progressively decreased until discontinuation.
Neonatal/infancy onset diabetes mellitus (NDM) is a monogenic form of diabetes occurring within 6 months from birth. NDM can be either permanent (PNDM), requiring lifelong treatment, or transient (TNDM), when insulin administration is necessary for 3 to 6 months and the remission persists. ${ }^{1}$ However, a significant number of patients with TNDM ( $\sim 50 \%$ of subjects) may relapse in late childhood or adolescence with type 2 diabetes (T2DM). NDM was considered extremely rare with an incidence of $1: 400,000$ to 1:500,000 live births, ${ }^{2}$ when it was defined as persistent hyperglycemia developing in the first 6 weeks of life in patients born at term and treated with insulin for at least 2 weeks. However, considering the new limit of 6 months of age the estimated incidence is $1 / 90,000$ to $1 / 210,000$ newborns. ${ }^{1,3}$ TNDM comprises $~ 50 \%$ of children with neonatal diabetes; ${ }^{4}$ a genetic diagnosis is made in up to $90 \%$ of these patients. The majority of cases $(\sim 70 \%)$ result from abnormalities of the imprinted region on chromosome 6q24, such as paternal uniparental isodisomy, paternally inherited duplication, or a methylation defect, leading to overexpression of paternally expressed genes. ${ }^{5}$ Most patients have intrauterine growth retardation at birth and failure to thrive. Other features noted in the neonatal period are macroglossia and umbilical hernia. ${ }^{6}$ Activating mutations in genes encoding the Kir6.2 (KCNJ11) and SUR1 (ABCC8) subunits of the pancreatic adenosine triphosphate (ATP)-sensitive $\mathrm{K}+$ received

September 7, 2017

accepted after revision

November 14, 2017
DOI https://doi.org/

$10.1055 / \mathrm{s}-0038-1636427$. ISSN 2157-6998.
Copyright $\odot 2018$ by Thieme Medical Publishers, Inc., 333 Seventh Avenue, New York, NY 10001, USA. Tel: +1(212) 584-4662.
License terms

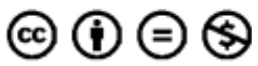


(K-ATP) channel account for $12 \%$ and $13 \%$ of cases of TNDM, respectively. ${ }^{7}$ We report the case of a preterm infant with TNDM due to a ABCC8 mutation.

\section{Case Presentation}

A white male was born at 30 weeks and 4 days of gestation by emergency cesarean section because of acute fetal distress and intrauterine growth retardation. The APGAR scores were 8 and 9 at 1 and 5 minutes, respectively. Birth weight was $1,065 \mathrm{~g}$ (10th percentile), length was $37 \mathrm{~cm}$ (11th percentile), and head circumference was $26 \mathrm{~cm}$ (6th percentile). He was the third baby born to nonconsanguineous parents. The infant's mother had gestational diabetes mellitus (GDM) requiring insulin treatment, resulting in good metabolic control. No family history of diabetes mellitus was referred. Physical examination at birth revealed no anomalies. In the second day of life, after a regular postnatal adaptation, our patients developed hyperglycemia, with glycemia varying from 170 to $351 \mathrm{mg} / \mathrm{dL}$, in absence of any stress, while the infant was receiving expressed breast milk in addition to parenteral nutrition, for a total glucose intake of $4.5 \mathrm{mg} / \mathrm{kg} / \mathrm{min}$. Full septic workup, including blood cultures, C-reactive protein (CRP), and urine culture, resulted negative. From the 5th day of life, the infant was treated with intravenous insulin boluses of $0.1 \mathrm{UI} / \mathrm{kg}$ (Humalog, Eli Lilly and Company, Indianapolis, IN), when glucose level rose over $250 \mathrm{mg} / \mathrm{dL}$, according to our protocol. On the 10th day of life, insulin and C-peptide plasma concentrations were determined and showed low values $(1.5 \mathrm{mU} / \mathrm{L}$ [normal range, 3-17], $0.32 \mathrm{nmol} / \mathrm{L}$ [normal range, 0.37-1.49], respectively); growth hormone was $20.1 \mathrm{U} / \mathrm{mL}$ (normal range, 0.05-4.5); thyroid hormones were normal; islet cell antibodies (ICA), insulin autoantibodies (IAA), antityrosine phosphatase antibodies (IA2), and antiglutamic acid decarboxylase (GADA) antibodies were negative; and abdomen ultrasound was normal. Due to the persistent hyperglycemia, and according to diabetologist advice, multiple daily injections (MDI) was started either in the abdomen or buttocks with insulin lispro $0.1 \mathrm{IU} / \mathrm{kg} /$ dose before meals, extemporaneously prepared by dilution in distilled water, without any effect reproducibility. On the 14th day of life, an attempt was made to administer regular insulin at the same dose and with the same dilution but without improvement. Furthermore, the newborn developed lipodystrophy in the injection areas. Continuous subcutaneous insulin infusion (CSII) was excluded because of lack of subcutaneous tissue. Considering the low weight and very poor insulin requirement $(\sim 0.15-0.20 \mathrm{UI} /$ dose $)$, we had to face the subsequent challenges: (1) lack of effect reproducibility, (2) operator-dependent effect, (3) need to use insulin syringes for injections, and (4) 8-mm syringe needles.

To overcome these obstacles, we had to plan an insulin delivery which was more reproducible and feasible also for the parents after discharge. To provide cartridges with diluted insulin lispro ready for use in pen injectors, we needed the Sterile Diluent ND-800 (Eli Lilly and Company,). This diluent is not authorized for commercial use in Italy and, therefore, it was necessary to activate the procedure to import abroad registered drugs. The following dilution was prepared by the hospital pharmacy: insulin lispro at a $1: 100$ dilution ( 1 IU diluted $=0.01$ IU insulin lispro) in sterile diluent to yield a concentration of $1 \mathrm{IU} / \mathrm{mL}$. A cartridge of $3 \mathrm{~mL}$ (297 units of sterile diluent added to $3 \mathrm{UI}$ of insulin lispro) was drawn up in the pen injector (HumaPen LUXURA HD, Eli Lilly and Company) allowing injections with a 4-mm needle. The insulin lispro dilution made it possible to administer accurate and exact insulin doses, thus resulting in good glycemic control. On the 66th day of life, which was the original due date, the infant was discharged from the hospital, with a daily insulin requirement of $0.15 \mathrm{UI} / \mathrm{kg}$. Weight gain during neonatal intensive care unit stay was poor, with a weight at term equivalent age of $2,076 \mathrm{~g}$ ( 0 percentile). A regular clinical follow-up was then performed: glycated hemoglobin (HbA1c) was measured quarterly and resulted in the normal range $(<5.8 \%$; $<40 \mathrm{mmol} / \mathrm{mol})$. Insulin was administered a few minutes before each breast feed, glucose level was checked 90 minutes after meals, and the values ranged between 100 and $150 \mathrm{mg} / \mathrm{dL}$, while fasting hyperglycemia persisted. After discharge, the infant's weight showed a marked catch up growth exceeding the 97th percentile. At 2 months, corrected age hypoglycemia occurred after meals and insulin doses were progressively decreased until suspension on the 133th day of life (2 months corrected age) ( - Fig. 1). At the time of writing this article, the patient is $\sim 22$ months old and is euglycemic without insulin treatment. He is being evaluated twice a year to check glucose metabolism since it is known that T2DM can occur around puberty in children with TNDM. Genetic DNA testing for neonatal diabetes revealed no genetic or epigenetic aberrations at the TNDM locus on chromosome 6q24. Therefore, next-generation sequencing (NGS) was performed and the mutation of c.2473C $>\mathrm{T}$ (p.Arg825Trp) in the $A B C C 8$ gene was identified. The mutation was inherited from the mother and is associated to neonatal diabetes ${ }^{4}$ (-Fig. 2). Genomic DNA extracted from peripheral lymphocyte was analyzed by NGS approach through targeted resequencing (454 FLX Roche platform) for a set of 182 genes. The sequencing array targets all coding exons and flanking sequences of all 14 known MODY genes, which were identified from genomewide linkage study or genome-wide association studies (GWAS) as increasing the risk of T2DM and causing diabetes in animal models. All identified genetic variants entered a systematic data analysis pipeline that included Sanger sequencing validation, segregation analysis, and a bioinformatic prediction of pathogenicity.

\section{Discussion}

Management of neonatal hyperglycemia is challenging and no universal guidelines are available. Stabilizing glucose levels and minimizing the hypoglycemic risk can be difficult and the initial approach is usually represented by intravenous insulin infusion. ${ }^{7}$ No insulin regimen, such as intermediate acting insulin, long-acting and short-acting insulin, or rapid-acting insulin analogue, has been demonstrated to be more effective than another. The need for tiny insulin doses and small body surface area with poor subcutaneous 


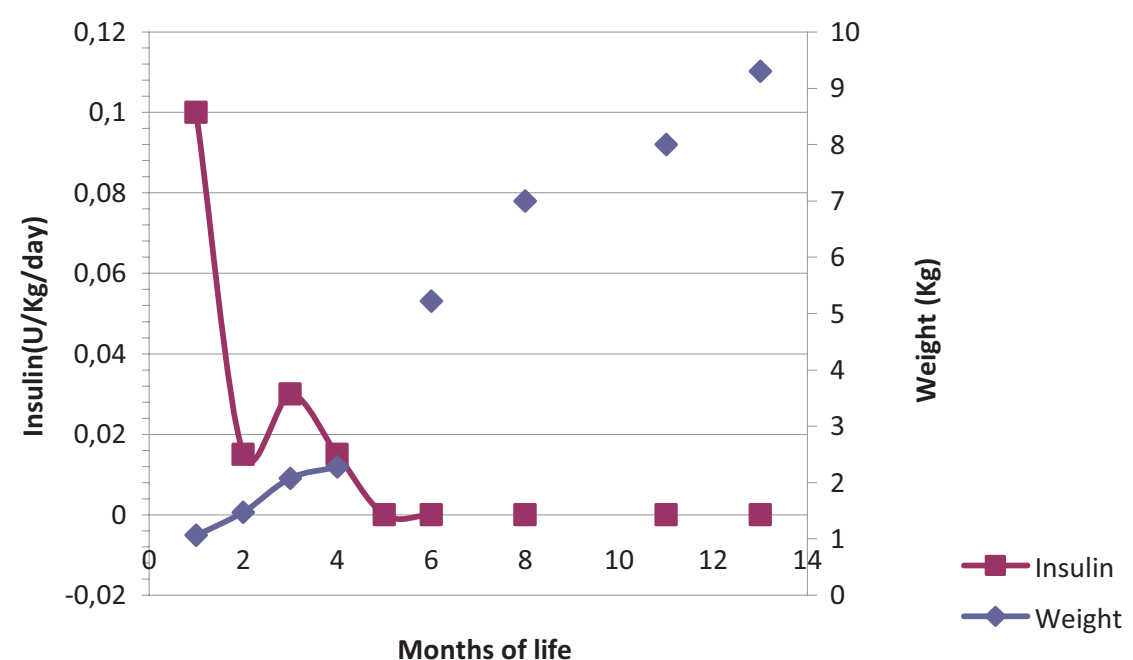

Fig. 1 Insulin need and weight timeline.

tissue represent limitations in preterm infants to the use of subcutaneous injections with pen injectors and syringes. Furthermore, breastfeeding is associated with intraindividual variation, requiring a very flexible insulin therapy and making it very difficult to establish the timing of insulin administration and the adequate insulin dose by carbohydrate counting. Moreover, repeated injections can lead to the development of lipodystrophy and to an erratic and unreliable insulin absorption, increasing the hypoglycemic risk in these patients who are already prone to hypoglycemia. CSII is

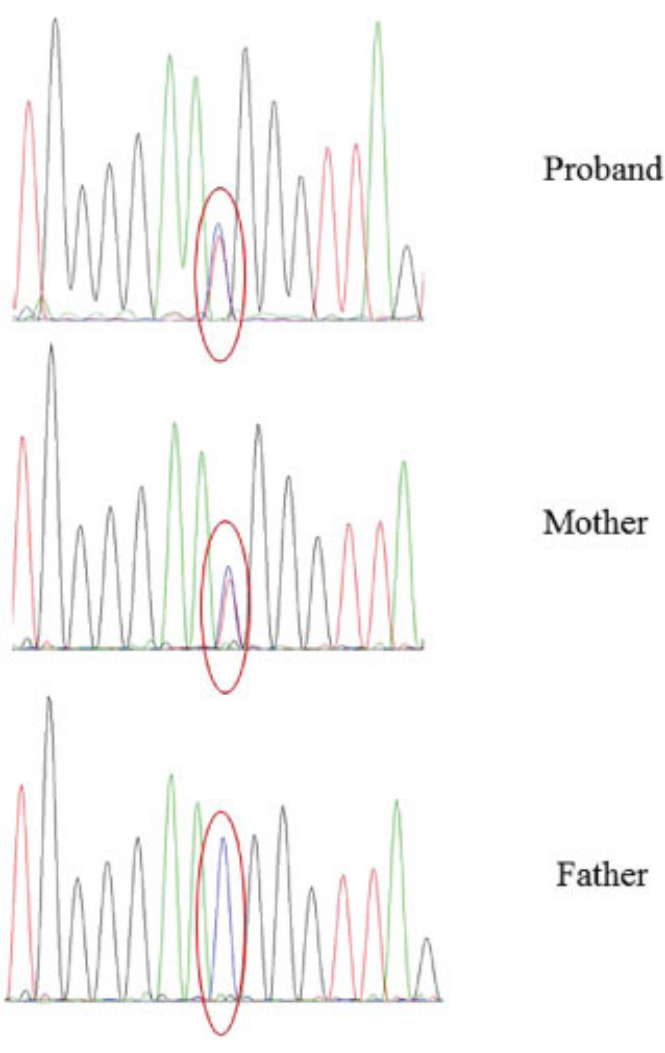

Fig. $2 A B C C 8$ Sanger sequencing in the proband and parents. considered safe and effective in obtaining adequate glycemic control in NDM and offers the possibility of precise dose titration and delivery of tiny insulin volumes. ${ }^{8,9}$

In this study, we report the case of a preterm infant with TNDM due to a $A B C C 8$ mutation inherited from the mother who experienced insulin-treated GDM. After delivery, the mother presented normal glucose metabolism, in particular, normal oral glucose tolerance test (OGTT) and HbA1c. ABCC8 mutations are associated with TNDM or PNDM, but more frequently are responsible for TNDM. The majority of ABCC8 mutations are de novo; however, dominant and recessive inheritance occurs as well. ${ }^{4}$ The $A B C C 8$ mutation leads to overactivation of K-ATP channels and impairment of insulin secretion from pancreatic $\beta$ cells by increasing the magnesium-nucleotide-dependent stimulatory action of SUR1 on the Kir pore. ${ }^{10}$ Patients with activating mutations of the KATP channel genes and PND, after initial insulin therapy, can be switched to sulfonylurea.

In our case, aforementioned clinical characteristics of our patients precluded the possibility of treating him with CSII, and moved us to use insulin lispro at a 1:100 dilution in sterile diluent. To our knowledge, this is the first patient successfully treated with a specifically prepared insulin dilution for pen injector instead of syringes with distilled water. The stabilization of glucose levels resulted in good weight gain and normal glucose metabolism without insulin and shows adequate growth and neurodevelopment. This case report sheds light on the importance of a multidisciplinary approach to complex cases: in this particular case, the neonatologist, the pediatric diabetologist, the pharmacist, the dietician, and the drug company were involved and cooperated to ensure our patient's therapeutic success.

Learning Points

- Neonatal/infancy onset diabetes mellitus (NDM) is a monogenic form of diabetes occurring within 6 months from birth that can be either permanent or transient.

- Management of neonatal hyperglycemia is particularly challenging in enteral fed very low birth weight infants. 
- This is the first case in which a patient with transient NDM is treated with a specifically prepared insulin dilution for pen injector instead of syringes with distilled water. This treatment allowed the stabilization of glycemia and normal growth.

\section{References}

1 Iafusco D, Massa O, Pasquino B, et al; Early Diabetes Study Group of ISPED. Minimal incidence of neonatal/infancy onset diabetes in Italy is 1:90,000 live births. Acta Diabetol 2012;49(05):405-408

2 Metz C, Cavé H, Bertrand AM, et al; NDM French Study Group. Neonatal diabetes mellitus. Neonatal diabetes mellitus: chromosomal analysis in transient and permanent cases. J Pediatr 2002; 141(04):483-489

3 Slingerland AS, Shields BM, Flanagan SE, et al. Referral rates for diagnostic testing support an incidence of permanent neonatal diabetes in three European countries of at least 1 in 260,000 live births. Diabetologia 2009;52(08):1683-1685
4 Flanagan SE, Patch AM, Mackay DJ, et al. Mutations in ATPsensitive $\mathrm{K}+$ channel genes cause transient neonatal diabetes and permanent diabetes in childhood or adulthood. Diabetes 2007;56(07):1930-1937

5 Vaxillaire M, Bonnefond A, Froguel P. The lessons of early-onset monogenic diabetes for the understanding of diabetes pathogenesis. Best Pract Res Clin Endocrinol Metab 2012;26(02):171-187

6 Temple IK, James RS, Crolla JA, et al. An imprinted gene(s) for diabetes? Nat Genet 1995;9(02):110-112

7 Karges B, Meissner T, Icks A, Kapellen T, Holl RW. Management of diabetes mellitus in infants. Nat Rev Endocrinol 2011;8(04):201-211

8 Beardsall K, Pesterfield CL, Acerini CL. Neonatal diabetes and insulin pump therapy. Arch Dis Child Fetal Neonatal Ed 2011;96 (03):F223-F224

9 Rabbone I, Barbetti F, Gentilella R, et al. Insulin therapy in neonatal diabetes mellitus: a review of the literature. Diabetes Res Clin Pract 2017;129:126-135

10 Babenko AP, Polak M, Cavé H, et al. Activating mutations in the ABCC8 gene in neonatal diabetes mellitus. N Engl J Med 2006;355 (05):456-466 